\title{
NDR1 functions as tumor suppressor in glioblastoma by phosphoralation of YAP
}

\section{Bin Chen ( $\sim 18553101842 @ 163 . c o m)$}

Peking University Third Hospital https://orcid.org/0000-0001-9588-3691

\section{Bin Liu}

Peking University Third Hospital

\section{Tao Yu}

Peking University Third Hospital

\section{Yunfeng Han}

Peking University Third Hospital

\section{Chao Wu}

Peking University Third Hospital

\section{Zhenyu Wang}

Peking University Third Hospital

\section{Research Article}

Keywords: NDR1, YAP, Hippo signaling pathway, glioblastoma

Posted Date: March 11th, 2021

DOl: https://doi.org/10.21203/rs.3.rs-276613/v1

License: (c) (1) This work is licensed under a Creative Commons Attribution 4.0 International License. Read Full License 


\section{Abstract}

Purpose: The NDR1(Nuclear Dbf2-related) kinase is a member of the NDR/LATS family which was a supplementary of Hippo pathway. However, whether NDR1 could inhibit glioblastoma (GBM) growth by phosphorylating YAP remains unknown. Meanwhile, the role of NDR1 in GBM was not clear. The purpose of this study was to investigate the role of NDR1-YAP pathway in GBM.

Materials and Methods: Bioinformation analysis and immunohistochemistry was performed to identify the expression of NDR1 in GBM. The effect of NDR1 on cell proliferation and cell cycle was analyzed utilizing cck-8, clone formation, immunofluorescence and flow cytometry respectively. In addition, the xenograft tumor model was established as well. Protein interaction was examined by Co-ip and immunofluorescence.

Results: Bioinformation analysis and immunohistochemistry of our petients' tumor tissues showed that expression of NDR1 in tumor tissue was relatively lower than that in normal tissues and was positively related to lower survival rate. NDR1 could markedly reduce the proliferation, colony formation of U87 and U251. Furthermore, the results of flow cytometry showed that NDR1 led to cell cycle arrest at the G1 phase. Tumor growth was also inhibited in xenograft nude mouse models in NDR1-OE group. Western blotting and immunofluorescence showed that NDR1 could integrate with and phophoralate YAP at S127 site. Meanwhile, NDR1 could mediate apopptosis process.

Conclusion: In summary, our findings pointed out that NDR1 functions as a tumor suppressor in GBM. NDR1 was identified as a novel regulator of YAP, which give us a in depth comprehension in Hippo signaling pathway.

\section{Introduction}

Glioma is the most common primary malignant brain tumor, which originates from glial cells or glial precursor cells. Glioblastoma (GBM, WHO grade IV) is one of the most common and most malignant primary brain tumors in the brain. Despite the current efforts of the medical investigations, the prognosis

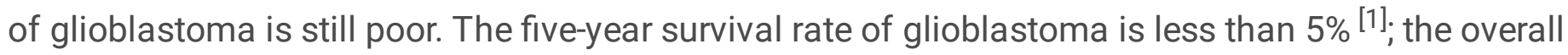
survival of patients in a row of clinical researches is between 14.6-26.3 months ${ }^{[2]}$. Therefore, how to improve the therapeutic effect of glioma is the focus and difficulty of neurosurgery research.

YAP was a transcriptional coactivator which could promote tissue and organ growth ${ }^{[3]}$. It was core effector of Hippo signaling pathway. MST1/2 could phosphoralate LATS1/2, which in turn phosphorelate YAP, leading to cytoplasmic retention and loss of function ${ }^{[4]}$. Accumulating evidence also showed that overexpression of YAP/TAZ was observed in many cancer types including glioma indicating that YAP/TAZ could not only promote organ growth, but also play a key role in tumor proliferation ${ }^{[5,6]}$. High YAP or TAZ activity enables the cell to escape contact inhibition and anoikis and to support anchorageindependent growth ${ }^{[7]}$. This result reminds us that regulation of YAP was significant for inhibition of 
glioma growth. At present, the upstream regulator of YAP has been under research. NDR1 came up into our sight because of its similarity to LATS.

NDR1 (Nuclear Dbf2-related, also known as serine/threonine kinase 38 or STK38) was a member of NDR family including NDR1, NDR2 (or STK38L), LATS1 (Large tumor suppressor kinases-1) and LATS2 (Large tumor suppressor-2), which is a member of the AGC group of the serine/threonine kinase family ${ }^{[8]}$. NDR protein kinase is highly conserved and is almost universally expressed in many different species ${ }^{[9]}$. NDR/LATS kinase is essential for cell growth, metabolism, proliferation and survival of many unicellular or multicellular organisms ${ }^{[10]}$. It is generally believed that the NDR has similar effects like LATS acting as tumor suppressor proteins in the Hippo pathway ${ }^{[11]}$. However, there is also evidence that NDR can also promote tumor growth. As an important supplement to the Hippo pathway, NDR1 has attracted much attention in the past ten years. Even though, the role of NDR1 in glioma still remains unknown. Now that NDR1 has similar function to LATS, it is also unclear whether NDR1 could exert tumor suppressing function by combining with YAP.

\section{Materials And Methods}

Bioinformatic analysis of ONCOMINE microarray database and SurvExpress database. The expression level of NDR1 (STK38) in glioblastoma was analyzed via ONCOMINE microarray datasets (https://www.oncomine.org). The datasets played a powerful role in screening differentially expressed genes (DEGs) between tumor and normal tissue. By searching "STK38", "Glioblastoma”, "Cancer vs Normal Analysis" and "mRNA", 5 datasets were acquired. The survival information of patients with lower and higher STK38 expression was obtained from SurvExpress: An Online Biomarker Validation Tool and Database for Cancer Gene Expression Data Using Survival Analysis

(http://bioinformatica.mty.itesm.mx/SurvExpress).

Cell culture. Human glioblastoma cancer cell lines U87 MG and U251 were purchased from the American Type Culture Collection (Manassas, VA, USA). U87 MG was cultured in MEM-a supplemented with 10\% fetal bovine serum (FBS, BI, Israel) and 1\% non-essential amino acid (NEAA, Gibco, USA). U251 was cultured in dulbecco's modified eagle medium (DMEM, Gibco, USA) supplemented with $10 \%$ fetal bovine serum (FBS, Bl, Israel). Cells were maintained at $37^{\circ} \mathrm{C}$ in a humidified incubator with a constant air flow of $5 \% \mathrm{CO} 2$.

Recombinant of lentivirus. The recombinant lentivirus overexpression (OE) RNA targeting NDR1 sequence (LV-OE-NDR1) and control sequence (Lv-Con) with green fluorescent protein (GFP) were purchased from Jikai (Shanghai, China). The mRNA sequence adopted in our study was NM_007271.4.

Cell infection of lentivirus. U87 MG and U251 cells were seeded in 6-well plates with the lentivirus; Cells were cultured in completed medium containing virus for 16 hours, then medium was replaced with normal medium. Observed by the fluorescence microscope, the observation of GFP-positive cells was 
represented as the infection efficiency. The culture medium containing puromycin (Solarbio, Beijing, China) was used to kill unsuccessfully transfected cells.

Western blot assay. U87 MG and U251 cells with stable NDR1 overexpression and control were collected and lysed in RIPA buffer (Solarbio, Beijing, China) and the western blot assay of equal volume lysate was performed as previously study described[18]. The following antibodies were used: anti NDR1 (1:500), pNDR1, YAP, p-YAP, MST1, LATS1, cleaved PARP and $\beta$-actin (all in 1:1000). For research of apopptosis, tumor necrosis factor-a(TNF-a, PEPRO TECH company, USA, Catalog\#:300-01A, 10ng/ml) was used to cells.

CCK-8 cell proliferation assay. U87 MG and U251 cells infected with Lv-OE-NDR1 or Lv-Con lentivirus were seed in 96-wells plates at a density of 2000 cells/well and incubated at $37^{\circ} \mathrm{C}$ culture chamber for $1,2,3,4$ or 5 days respectively. Then the CCK-8 solution (Dojindo, Japan) was added into the culture medium, and the cells infected with virus were incubated with above medium for $2 \mathrm{~h}$ at $37^{\circ} \mathrm{C}$ in humidified $95 \%$ air and $5 \%$ CO2. The optical density (OD) was measured at 450nm using a Microplate Reader (Bio-Rad Laboratories Inc, Hercules, CA, USA). Each group had five duplicate and the experiment was performed in triplicate.

Colony formation assay. Cells infected with Lv-OE-NDR1 or Lv-Con lentivirus were seeded in $10 \mathrm{~cm}$ dishes (U87: 800/well; U251: 1000/well, respectively). The supernatant was discarded and culture medium was replaced every 3 days. After cultured 14 days, the cells were washed with PBS two times and fixed with $4 \%$ paraformaldehyde for 30 minutes. In the last, after washed with PBS again, the colonies were stained with $5 \%$ crystal violet for 30 minutes. The number of colonies (> 50 cells/colony) was count using Image $J$. Each group had three repetitions and the experiment was performed three times.

Cell cycle analysis. The infected U87 MG and U251 cells were seed in 6-well dishes, then cells were collected by trypsinization and fixed with $75 \%$ cold ethanol for $24 \mathrm{~h}$ at $4{ }^{\circ} \mathrm{C}$. After washed with cold PBS two times, cells were resuspended with 500ul RNaseA (KeyGEN Bio Tech company, Jiangsu, China) at 37 ${ }^{\circ} \mathrm{C}$ for $30-60$ minutes. Then, cells were stained with 500ul propidium iodide (KeyGEN Bio Tech company, Jiangsu, China) at room temperature for 60 minutes in the dark. Cell cycle distribution was recorded with a flow cytometer (BD Bioscience, USA). The experiment was performed according to the manufacture's instruction in triplicate.

In vivo xenograft assay. Female nude mice were purchased from Model Animal Research Center of Peking University Health and Science Center and housed in specific pathogen-free barrier facilities. Twenty four 6 -week-old female nude mice were randomly divided into two groups. A total of $1 \times 10^{7}$ U87 cells infected with Lv-OE-NDR1 were injected into the right axillary of 12 mice, and other 12 mice were injected with U87 cells infected with Lv-Con in equal concentration. The diameters of xenograft were measured using a slide caliper every 3 days for 32 days. The xenograft tumor volume was calculated using the formula: $V=\left(\right.$ length $\left.\times w_{\text {width }}{ }^{2}\right) / 2$. At the end of the experiment, mice were euthanized and imaged. 
The xenograft tumors were removed from each mouse body, imaged, weighed, and fixed in $4 \%$ paraformaldehyde.

Immunohistochemistry $(I H C)$ staining. The nude mouse xenograft tumors were paraffin embedded and sliced, then these tumor tissues were subjected to IHC staining as previously study described. Anti-NDR1 primary antibody (1: 50, Santa cruze, USA), Ki-67 antibody ( $1 ; 200$, Cell Signaling Technology, USA) and hematoxylin-eosin (HE) staining was performed. Patients' tumors acquired from surgery were embedded and sliced. Anti-NDR1 primary antibody (1: 50, Santa cruze, USA) and anti-YAP antibody (1:100, Abcam, USA) were used.

Immunofluorescence staining. The infected U87 MG and U251 cells were seed in confocal dishes. After washed with PBS 3 times, cells were resuspended with 500ul formaldehyde at toom temperature for 5 minutes. Then, cells were sealed by $2 \%$ goat serum at room temperature for 30 mins and incubated with Anti-NDR1 primary antibody (1: 100, Santa cruze, USA) and anti-YAP antibody (1:200, Abcam, USA) and DAPI. In order to improve kinase activity of p-NDR1, Okadaic acid (OA, Cas:78111-17-8, APEXBIO company, Houston, USA, dose: 10nM) was used.

Terminal Deoxynucleotidyl Transferase Deoxyuridine Triphosphate Nick-End Labeling Assay (TUNEL). Xenograft tumors were fixed with $4 \%$ paraformaldehyde for $20 \mathrm{~min}$ at room temperature and rinsed with PBS. After that, slides were incubated with $0.1 \%$ Triton X-100 solution for 2 min on ice and rinsed twice with PBS. Next, $50 \mu \mathrm{l}$ TUNEL reaction mixture was added on slides and incubated in a humidified atmosphere for $1 \mathrm{~h}$ at $37^{\circ} \mathrm{C}$ in dark. After that, cells were rinsed with PBS and stained with Hoechst 33258. Finally, cells were analyzed under a fluorescence microscope.

Statistical analysis. Dates were presented as mean \pm SD form at least three calculations. Chi-square test was employed in statistical comparation between low expression NDR1 patients and high expression NDR1 patients. For comparison of treatment groups, we performed unpaired t-tests (Mann-Whitney), oneway or two-way ANOVA (where appropriate). In addition, the Kaplan-Meier analysis was employed to evaluate the correlation between prognosis of GBM patients and NDR1 mRNA levels in our sample. The Statistical Package for Social Science version 21 (SPSS Inc. Chicago, IL, USA) and GraphPad Prim 5 software (GraphPad Software Inc, Sam Diego, CA, USA) were used to calculate the statistics and $p<0.05$ was considered statistically significant.

\section{Results}

\section{NDR1 is downregulated in glioblastoma tissues and is negatively associated with clinical outcomes}

To investigate the role of NDR1 in GBM, we measured its expression level in an GBM tissues and normal tissues we got from pathology department. The expression level of NDR1 was relatively lower in GBM tumor tissues than that of normal tissues (Figure 1A,B). Meanwhile, we gathered general information of patients including age, gender, tumor size, tumor extension, type of surgery and radiation therapy (table 1). Analysis suggested that the expression level of NDR1 was decreased in big tumors compared to small 
tumors, indicating that NDR1 has a negative correlation with tumor size (table 2). survival analysis also suggested that patients with lower NDR1 levels had shorter overall survival times (Figure 1C). Moreover, data from Oncomine showed that NDR1 levels were lower in GBM tissues than in normal tissues (Figure 1D-F). Further, data from SurvExpress (Brain-Gravendeed French Glioblastoma GSE16011) also showed that higher expression represents lower risk and lower expression represents higher risk. Patients who had lower expression showed tendency of poor overall survival (Figure 1G, H). Collectively, these results showed that NDR1 expression levels are low in GBM patients. Lower expression of NDR1 is associated with clinical prognosis and tumor size.

\section{NDR1 was upregulated in U87 and U251 cells after transfection of lentivirus}

To investigate the role of NDR1 in GBM, we transfected it with lentivirus-mediated OE-RNA in U87 and U251 cells. The infection efficiency of GBM cancer cells were identified by GFP expression (Fig. 2A). The protein and mRNA levels of NDR1 in U87 and U251 cells transfected with lentivirus-mediated OE-RNA were significantly upregulated compared to cells infected with control (Fig. 2B,C). These results indicated that the expression of NDR1 was promoted successfully.

\section{NDR1 decreased cell proliferation in vitro}

Cell proliferation is an important indicator to evaluate cell activity, metabolism, physiological and pathological conditions. Based on above findings, we further investigated the tumor suppressor effect of NDR1 in glioblastoma. As is shown in figure 3, cck-8 results showed that NDR1-overexpression in both U87 and U251 significantly decreased tumor viability (Figure 3-A, B). After 14 days incubation, the number of clones of NDR1-overexpression cells was significantly less than NDR1-control cells (Figure 3-C-F). EdU (5-Ethynyl-2'-deoxyuridine) is widely used in cell proliferation, cell differentiation, growth and development. DNA replication activity can be directly and accurately detected based on the specific reaction between Apollo ${ }^{\circledR}$ fluorescent dye and EdU. Our EDU fluorescent images showed that U87-control group had a more active proliferation ability (Figure 3-G-J). Besides, cell cycle analysis suggested that U87-overexpression group were dramatically arrested in G1 phase compared to control, and the number of cells in the S and G2 phase were decreased. Similar results were also found in U251 (Figure 3-K-M). Western blotting was used to further confirm the cell cycle arresting. Western blotting results showed that cyclin-E1 was down-regulated in OE group (Figure 3-N, O). Cell migration and invasion were also performed (supplementary figure S1-A, B). Results turned out to be that NDR1 barely affected migration and invasion. EMT pathway was detected by western blotting. The results showed that NDR1 has little effects on EMT pathway (supplementary figure S1-C).

\section{NDR1 inhibited tumor growth in vivo}

To further validate the function of NDR1 in glioblastoma tumorigenesis in vivo, U87 cells infected with LvOE-NDR1 or LV-Control were injected subcutaneously to the six-week-old nude mouse respectively. The tumor sizes and weights were measured after nude mice sacrificed. As shown in the figure 3 , the tumor size and weight of nude mice injected with Lv-OE-NDR1 infection cells were significantly lower than mice 
injected with control (Figure 4-A, B). The exnografts were given HE staining to demonstrate that our exnografts were glioblastoma tumors which originated from U87 cells (Supplementary figure S2-A). Furthermore, the IHC indicated that expression of NDR1 in overexpression tumors were higher than control (Figure 4-C,H, Figure s2-B). The expression of ki-67 in overexpression tumors were lower than control (Figure 4-D,I, Figure s2-C). Besides, TUNEL images also showed that NDR1 overexpressing promoted apoptosis process (Figure 4-E,J, Figure s2-D). Both the tumor volume and tumor weight were significantly reduced in OE group (Figure 4-F,G). These results demonstrated that NDR1 upregulation inhibited the tumorigenicity of glioblastoma cells in vivo.

\section{NDR1 could combine with YAP and phosphorylate YAP}

We wonder how NDR1 plays its tumor suppressor role in GBM. Considering that NDR1 and LATS belongs to same AGC family, we hypothesized that NDR1 might also interact with YAP. To investigate interaction between NDR1 and YAP, inmunofluorence was done. U87 was transfected with NDR1-OE plasmids with mscarlet. Inmunofluorence was performed when mscarlet was seen under microscope. Immunofluorence microscopy showed that NDR1 and YAP can co-localize in human cells (Figure-5A, supplementary figures3). To further verify the interaction between NDR1 and YAP, CO-IP was confirmed. We co-transfected NDR1-mscarlet and Flag-tagged YAP into U87 cells. Immunoprecipitation with anti-Flag antibody showed that NDR1 was co-immunocipitated with YAP. To rule out overexpression artifacts, we examined the interaction between endogenous NDR1 and YAP, showing that YAP co-immunocipitates NDR1 (Figure-5B). This result identified that NDR1, apart from LATS1/2, could interact with YAP. Post-translational modifications including phosphorylation and acetylation are critical for the protein stability and functional activity of YAP. Therefore, we explored whether upregulated NDR1 could increase YAP stability by modulating YAP phosphorylation. We found that NDR1 could phosphorylate YAP at S127 site (Figure$5 C-D)$.

\section{NDR1 regulates the localization and transcriptional activity of YAP}

Considering that phosphorylation of YAP can result in the inactivation of YAP by cytoplasmic retention, we hypothesized that NDR1 overexpression might reduce nuclear YAP1 levels. To test this hypothesis, we performed immunofluorescence. Phosphorylation of YAP is regarded to promote YAP cytoplasmic localization and inactivation. Okadaic acid (OA) was a kind of phosphatase inhibitors. Studies showed that OA causes a global increase in phosphorylation level including level of p-NDR1. When added OA to U87 cells, more YAP localized in the cytoplasm in NDR1-overexpression group than control group (Figure$6 \mathrm{~A}$ ). The ratio of nuclear versus cytoplasmic YAP1 was significantly reduced in cells overexpressing NDR1 compared to controls (Figure-6B).

\section{NDR1 could mediate apopptosis of TNF-a stimulation}

Due to the TUNEL staining results suggesting that the apopptosis degree was significantly higher in NDR1-OE group than that of NDR1-ctrl group, we did further research on the role of NDR1 in apopptosis in U87 cells by western blotting. As is shown in figure 7, results showed that after TNF-a stimulation for 4 
hours, the expression of cleaved PARP was higher in NDR1-OE group than that of NDR1-ctrl group with or without TNF-a and $\mathrm{H}_{2} \mathrm{O}$.

\section{Role of NDR1 in Hippo signaling pathway}

Together with previous findings, our results indicated that NDR1, as a supplementary of Hippo signaling pathway, plays a tumor suppressor role in GBM by phosphorylating YAP (Figure-8-A). besides, NDR1 could also mediate apopptosis process (Figure-8-B).

\section{Discussion}

YAP was found to be an oncogene in GBM, which was the most common and popular malignant glioma with a survival of 15 months $^{[1]}$. Researchers found that YAP is highly expressed in gliomas and be able to promote tumor cell proliferation and invasion in glioma which means regulation of YAP is critical for tumor proliferation ${ }^{[12,13]}$. YAP is the downstream core effector molecule of the Hippo pathway which was first discovered in Drosophila and has the functions of regulating organ growth, development, proliferation, and apoptosis ${ }^{[5,14]}$. A large number of studies have found that the Hippo pathway is closely related to the occurrence of many malignant tumors ${ }^{[5,15]}$. In many malignant tumors, the Hippo pathway is abnormal, resulting in increased non-phosphorylated YAP content. As a core effector molecule, YAP is located in the most downstream of the Hippo pathway, and its upstream has MST1/2, LATS1/2 and other kinases. MST1/2 can phosphorylate LATS1/2 and LATS1/2 can phosphorylate YAP, making it inactive, staying in the cytoplasm, and unable to enter the nucleus to function.

NDR1/2 was speculated to be a tumor suppressor like LATS1/2 in Hippo pathway because their similarities. NDR and LATS belong to NDR/LATS family and both of them need to be phophorylated on two residues to achieve full kinase activation just like other AGC kinases ${ }^{[16]}$. Notably, all NDR/LATS kinases share two unique characteristics including a conserved N-terminal regulatory domain (NTR) in proximity to the catalytic domain and an insertion between subdomains VII and VIII of the catalytic domain ${ }^{[17]}$. Both of these two domains play roles in the regulation of NDR/LATS kinases. Study also showed that the NDR/LATS family, including NDR, LATS, Orb6 and Cot-1, have same sequences of phosphorylation sites corresponding to both Ser-281 and Thr-444 which means NDR/LATS family is highly conserved ${ }^{[18]}$. As we all know, NDR1/2 play its role through phosphorelation of two sites, Ser-281 and Thr-444. Thus, it is speculated that NDR/LATS family might have similar functions. Studies also showed that Ste20-like Hippo kinases was shown to activate the fly NDR/LATS kinase Warts ${ }^{[19]}$. Specifically, NDR1/2 could be phosphorylated by MST1/2 on their Thr residue located in HM and regulated by MOB1. In this regard, NDR1/2 could be considered as a novel member of Hippo pathway. Consequently, we speculated that NDR1/2 may play a role as tumor suppressor.

Many studies have shown that NDR1/2 has a tumor suppressor effect. Cui et al. found that the expression of NDR1 in cancer tissues of patients with gastric cancer was significantly lower than that of normal gastric mucosa through gene chip research. He proposed that the expression level of NDR1 can 
be used for early warning of gastric cancer ${ }^{[20]}$. In a chemically induced mouse skin cancer model, $L$ Hummerich used gene expression analysis to find that compared with normal skin tissue, the expression of NDR2 in skin tumor specimens was significantly reduced. The author also carried out immunofluorescence to verify the result which is indeed the case. Finally, using q-PCR analysis, it was found that cancer-promoting factors were significantly increased, but the expression of NDR2 was significantly reduced ${ }^{[21]}$. Monica Messina et al. conducted an oligonucleotide array to study the PK gene expression profile in adult acute lymphoblastic leukemia (ALL) samples and found that the expression of NDR1 was significantly reduced in ALL ${ }^{[22]}$. A recent study by Zhang et al. found that ${ }^{[23]}$ the intestinal epithelial cells of Ndr1 null mice were conditionally knocked out of NDR1/2, and then azoxymethane/dextran sodium sulfate enema was used to observe whether they induced colon cancer. The results showed that there were fewer nodules in the colon of normal control mice, but more than ten nodules in the colon of NDR1/2 cKO mice, indicating that NDR1/2 has an anti-tumor effect.

However, NDR has also been found to be elevated in some tumors. Adewale Adeyinka et al. found that compared to ductal carcinoma in situ (DCIS) without necrosis, the mRNA expression level of NDR1 was higher in DCIS with necrosis, but did not reach a statistically significant difference ${ }^{[24]}$. Besides, the high expression of NDR1 is also closely related to the proliferation of B-cell lymphoma ${ }^{[25]}$. Brygida C. Bisikirska et al. found that in human B-cell lymphoma, NDR1 affects the stability of MYC protein in a kinase activity-dependent manner. When NDR1 is silenced, the stability of MYC protein and the survival rate of lymphoma cells are significantly reduced. And NDR1 can regulate the transcription activity of MYC. Moreover, in order to evaluate the effect of NDR1 knockdown on the tumorigenic growth of MYCdependent human lymphomas in vivo, the authors introduced xenografts of stable cell lines of NDR1 shRNA into SCID mice. The authors found that tumor growth was delayed by 8 days in mice using NDR1 shRNA. It shows that NDR1 knockdown inhibited tumor growth ${ }^{[25]}$. Taken together, NDR1 is a versatile molecule that plays a differential role in different cancer progression. However, its role in glioma remains unknown. This study suggested that NDR1 could inhibit tumor proliferation ability and has little effect on migration or invasion.

As the upstream regulator of YAP, LATS1/2 is considered a tumor suppressor gene. For a long time, LATS kinase has been established as the only regulator of YAP and TAZ ${ }^{[26,27]}$. There is no evidence that NDR1/2 can also mediate the phosphorylation of YAP ${ }^{[5,23]}$. 10 years ago, Hao, Y. et al. found that NDR1/2 cannot interact with YAP. Therefore, he believed that NDR1/2 is not a kinase of YAP in mammals. But Hao, Y. et al. did not continue to do more in-depth research on NDR1/2. And other studies have shown that the decrease of NDR1/2 level is related to the increase of TAZ activity, but the direct link between NDR1/2 and TAZ has not yet been established ${ }^{[16]}$. In recent years, Zhang et al. ${ }^{[23]}$ came up with a new conclusion. He use azoxymethane/Portugal Glycan sodium sulfate enema to stimulate intestinal epithelial cell of Ndr1 null mice which were conditionally knocked out of NDR1/2 to investigate whether it could induce colon cancer. Results showed that there were fewer nodules in the colon of normal control mice, but more than ten nodules in the colon of NDR1/2 cKO mice, indicating that NDR1/2 has an antitumor effect. Furthermore, the author found that after knocking out NDR1/2, the expression level of non- 
phosphorylated YAP was significantly increased, and the expression level of p-YAP was significantly reduced, indicating that NDR can play an anti-tumor proliferation effect through phosphorylation of YAP. His conclusion was summarized from mice. We want to observe NDR1/2-YAP interaction from human beings. This study suggested that NDR1 could phophorylate YAP at S127 site to play its role in mammalian cells. Moreover, our study found that NDR1 could interact with YAP directly to play its role which overthrow Hao, Y's point of view. Our results suggested that LATS was not the only upstream regulator of YAP which was a significant supplementary for Hippo pathway.

Besides, NDR1 could also mediate apoptosis process to play its tumor suppressor role. Apoptosis is an important form of maintaining homeostasis and inhibiting tumor formation. In cancer cells, it is often suppressed by the inactivation of corresponding tumor suppressor factors ${ }^{[28]}$. It has been reported that reintroduction of RASSF1A can induce cell cycle inhibition by inactivating the APC/Cdc20 complex [29]. Studies have shown that Fas and TNF-a receptors are stimulated by promoting the phosphorylation of the NDR1/2 hydrophobic motif (Thr444/442). In addition, NDR1/2 is necessary for Fas receptor-induced apoptosis. Fas receptor stimulation promotes the direct phosphorylation of MST1 and activation of NDR1/2. In addition, the apoptosis induced by RASSF1A largely depends on the presence of NDR1/2 [30] Hauke Cornils et al. found that in NDR1-deficient mice, the expression of NDR1 can be activated after endogenous and exogenous pro-apoptotic stimuli are given, indicating that NDR1 plays an important role in regulating cell apoptosis.

Since YAP has been known as a biomarker of GBM, it is beneficial for patients to investigate what molecular can phosphorelate YAP. Our data suggest that NDR1 inhibit GBM progression by phophorelation of YAP. We found that YAP could bind to NDR1 and be phosphorelated into p-YAP at S127. Besides, when NDR1 was overexpressed, more non-phosphorylated YAP located in the cytoplasm. The effect of NDR1 on phosphorelating YAP provides us a potential novel target for glioma therapy. This discovery is very important for the study of the Hippo pathway, which also determined that NDR1/2 can be treated as an important supplement to the Hippo pathway. Our study also indicats that the Hippo pathway does not phosphorelate YAP simply through LATS. NDR1 may have a more important role than LATS1/2.

\section{Conclusion}

In summary, our findings pointed out that NDR1 functions as a tumor suppressor in glioblastoma. NDR1 was identified as a novel regulator of YAP, which give us a new in depth comprehension in Hippo signaling pathway.

\section{Declarations}

\section{Acknowledgement}


We thank Zhenyu Wang for teaching me, we thank Yunfeng Han and Chao Wu for the advices for writing this article. We thank Bin Liu and Tao Yu for supplying the clinical data.

\section{Author Contribution statement}

Bin Chen and Bin Liu: Design project ideas and implement experiments.

Bin Chen wrote this main manuscript text.

Yunfeng Han, Tao Yu and Chao Wu helped to collect specimens.

Zhenyu Wang guided Bin Chen to write this manuscript.

\section{Disclosure Statement}

The authors have no conflict of interest or financial ties to disclosure.

\section{Ethical approval}

Our research was approved by the Medical Science Research Ethics Committee of our hospital and followed the guidance of WMA Declaration of Helsinki-Ethical Principles for Medical Research Involving Human Subjects. Considering our specimens are all obtained from the pathology department, the ethics committee believes that our research does not require additional patient informed consent, and only needs to pay attention not to leak patients' information. As a result, there is no relevant ethical ID.

\section{Data availability statement}

I confirm that the data that support the findings of this study are available in ONCOMINE database and SurvExpress database. These data were derived from the above database. The specific operation method has been introduced in the manuscript. By searching "STK38", "Glioblastoma", "Cancer vs Normal Analysis", 5 datasets were acquired. The survival information of patients with lower and higher STK38 expression was obtained from SurvExpress: An Online Biomarker Validation Tool and Database for Cancer Gene Expression Data Using Survival Analysis (http://bioinformatica.mty.itesm.mx/SurvExpress). Data originated from Brain-Gravendeed French Glioblastoma GSE16011.

\section{Funding}

This study was supported by grants from the Capital Clinical Characteristic Application Research Project initiated by Beijing Science and Technology Commission (B70489-01). The funders had no role in the study design, data collection and analysis, decision to publish, or preparation of the manuscript.

\section{References}

1.Ostrom QT, Bauchet L, Davis FG, Deltour I, Fisher JL, Langer CE, et al. The epidemiology of glioma in adults: a "state of the science" review. Neuro Oncol. 2014 Jul;16(7):896-913. doi: 
2.Stupp R, Hegi ME, Gorlia T, Erridge SC, Perry J, Hong YK, et al. Cilengitide combined with standard treatment for patients with newly diagnosed glioblastoma with methylated MGMT promoter (CENTRIC EORTC 26071-22072 study): a multicentre, randomised, open-label, phase 3 trial. Lancet Oncol. 2014 Sep;15(10):1100-8. doi: 10.1016/S1470-2045(14)70379-1.

3.Zhao B, Li L, Lei Q, Guan KL. The Hippo-YAP pathway in organ size control and tumorigenesis: an updated version. Genes Dev. 2010 May;24(9):862-74. doi: 10.1101/gad.1909210.

4.Hong W, Guan KL. The YAP and TAZ transcription co-activators: key downstream effectors of the mammalian Hippo pathway. Semin Cell Dev Biol. 2012 Sep;23(7):785-93. doi:

10.1016/j.semcdb.2012.05.004.

5.Yu FX, Zhao B, Guan KL. Hippo Pathway in Organ Size Control, Tissue Homeostasis, and Cancer. Cell. 2015 Nov 5;163(4):811-28. doi: 10.1016/j.cell.2015.10.044.

6.Orr BA, Bai H, Odia Y, Jain D, Anders RA, Eberhart CG. Yes-associated protein 1 is widely expressed in human brain tumors and promotes glioblastoma growth. J Neuropathol Exp Neurol. 2011 Jul;70(7):56877. doi: 10.1097/NEN.0b013e31821ff8d8.

7.Zhao B, Wei X, Li W, Udan RS, Yang Q, Kim J, et al. Inactivation of YAP oncoprotein by the Hippo pathway is involved in cell contact inhibition and tissue growth control. Genes Dev. $2007 \mathrm{Nov}$ 1;21(21):2747-61. doi: 10.1101/gad.1602907.

8.Manning G, Whyte DB, Martinez R, Hunter T, Sudarsanam S. The protein kinase complement of the human genome. Science. 2002 Dec 6;298(5600):1912-34. doi: 10.1126/science.1075762.

9. Hergovich A, Stegert MR, Schmitz D, Hemmings BA. NDR kinases regulate essential cell processes from yeast to humans. Nat Rev Mol Cell Biol. 2006 Apr;7(4):253-64. doi: 10.1038/nrm1891.

10.Geng,W.; He, B.;Wang, M.; Adler, P.N. The tricornered gene, which is required for the integrity of epidermal cell extensions, encodes the Drosophila nuclear DBF2-related kinase. Genetics 2000, 156, 1817-1828.

11.Zallen JA, Peckol EL, Tobin DM, Bargmann Cl. Neuronal cell shape and neurite initiation are regulated by the Ndr kinase SAX-1, a member of the Orb6/COT-1/warts serine/threonine kinase family. Mol Biol Cell. 2000 Sep;11(9):3177-90. doi: 10.1091/mbc.11.9.3177.

12.Wang $Y$, Pan $P$, Wang $Z$, Zhang Y, Xie $P$, Geng $D$, et al. $\beta$-catenin-mediated YAP signaling promotes human glioma growth. J Exp Clin Cancer Res. 2017 Sep 29;36(1):136. doi: 10.1186/s13046-017-0606-1.

13. Zhang $Y$, Xie $P$, Wang $X$, Pan $P$, Wang $Y$, Zhang $H$, et al. YAP Promotes Migration and Invasion of Human Glioma Cells. J Mol Neurosci. 2018 Feb;64(2):262-272. doi: 10.1007/s12031-017-1018-6. 
14.Huang J, Wu S, Barrera J, Matthews K, Pan D. The Hippo signaling pathway coordinately regulates cell proliferation and apoptosis by inactivating Yorkie, the Drosophila Homolog of YAP. Cell. 2005 Aug 12;122(3):421-34. doi: 10.1016/j.cell.2005.06.007.

15.Pan D . The Hippo Signaling Pathway in Development and Cancer[J]. Developmental Cell, 2010, 19(4):0-505.

16. Hergovich A. The Roles of NDR Protein Kinases in Hippo Signalling. Genes (Basel). 2016 May 18;7(5):21. doi: 10.3390/genes7050021.

17.Pearce LR, Komander D, Alessi DR. The nuts and bolts of AGC protein kinases. Nat Rev Mol Cell Biol. 2010 Jan;11(1):9-22. doi: 10.1038/nrm2822.

18.Millward TA, Hess D, Hemmings BA. Ndr protein kinase is regulated by phosphorylation on two conserved sequence motifs. J Biol Chem. 1999 Nov 26;274(48):33847-50. doi:

$10.1074 / j b c .274 .48 .33847$.

19.Wu S, Huang J, Dong J, Pan D. hippo encodes a Ste-20 family protein kinase that restricts cell proliferation and promotes apoptosis in conjunction with salvador and warts. Cell. 2003 Aug 22;114(4):445-56. doi: 10.1016/s0092-8674(03)00549-x.

20.Cui DX, Zhang L, Yan XJ, Zhang LX, Xu JR, Guo YH, et al. A microarray-based gastric carcinoma prewarning system. World J Gastroenterol. 2005 Mar 7;11(9):1273-82. doi: 10.3748/wjg.v11.i9.1273.

21. Hummerich L, Müller R, Hess J, Kokocinski F, Hahn M, Fürstenberger G, et al. Identification of novel tumour-associated genes differentially expressed in the process of squamous cell cancer development. Oncogene. 2006 Jan 5;25(1):111-21. doi: 10.1038/sj.onc.1209016.

22.Messina M, Chiaretti S, Tavolaro S, Peragine N, Vitale A, Elia L, et al. Protein kinase gene expression profiling and in vitro functional experiments identify novel potential therapeutic targets in adult acute lymphoblastic leukemia. Cancer. 2010 Jul 15;116(14):3426-37. doi: 10.1002/cncr.25113.

23.Zhang L, Tang F, Terracciano L, Hynx D, Kohler R, Bichet S, et al. NDR functions as a physiological YAP kinase in the intestinal epithelium. Curr Biol. 2015 Feb 2;25(3):296-305. doi: 10.1016/j.cub.2014.11.054.

24.Adeyinka A, Emberley E, Niu Y, Snell L, Murphy LC, Sowter H, et al. Analysis of gene expression in ductal carcinoma in situ of the breast. Clin Cancer Res. 2002 Dec;8(12):3788-95.

25.Bisikirska BC, Adam SJ, Alvarez MJ, Rajbhandari P, Cox R, Lefebvre C, et al. STK38 is a critical upstream regulator of MYC's oncogenic activity in human B-cell lymphoma. Oncogene. 2013 Nov 7;32(45):5283-91. doi: 10.1038/onc.2012.543.

26.Oh H, Irvine KD. Yorkie: the final destination of Hippo signaling. Trends Cell Biol. 2010 Jul;20(7):410-7. doi: $10.1016 /$ j.tcb.2010.04.005. 
27. Meng Z, Moroishi T, Guan KL. Mechanisms of Hippo pathway regulation. Genes Dev. 2016 Jan 1;30(1):1-17. doi: 10.1101/gad.274027.115.

28.Debatin K M , Krammer P H . Death receptors in chemotherapy and cancer[J]. Oncogene, 2004, 23(16):2950-2966.

29.Song MS, Song SJ, Ayad NG, Chang JS, Lee JH, Hong HK, et al. The tumour suppressor RASSF1A regulates mitosis by inhibiting the APC-Cdc20 complex. Nat Cell Biol. 2004 Feb;6(2):129-37. doi:

10.1038/ncb1091.

30.Vichalkovski A, Gresko E, Cornils H, Hergovich A, Schmitz D, Hemmings BA. NDR kinase is activated by RASSF1A/MST1 in response to Fas receptor stimulation and promotes apoptosis. Curr Biol. 2008 Dec 9;18(23):1889-95. doi: 10.1016/j.cub.2008.10.060.

\section{Tables}


Table 1. 158 patients' general information

\begin{tabular}{ll}
\hline Variables & Percentage (\%) \\
\hline Age & $59.33 \pm 13.67$ \\
Sex & \\
Female & $56(35.4 \%)$ \\
Male & $102(64.6 \%)$ \\
Tumor size,mm, number & \\
$\leq 20 m m$ & $20 \square 12.7 \% \square$ \\
$21-40 m m$ & $57 \square 36.1 \% \square$ \\
$41-60 m m$ & $56 \square 35.4 \% \square$ \\
$\square 60 m m$ & $25 \square 15.8 \% \square$ \\
Tumor extension, number & \\
Localized at one side, not cross the mid line & $131 \square 82.9 \% \square$ \\
Invasive,cross the mid line and distal metastasis & $27 \square 17.1 \% \square$ \\
Type of surgery, number & \\
Partial resection & $32 \square 20.3 \% \square$ \\
Gross resection & $60 \square 38 \% \square$ \\
Radical resection & $66 \square 41.8 \% \square$ \\
Radiation therapy & $123(77.8 \%)$ \\
\hline Nnknown & $22(13.9 \%)$ \\
\hline
\end{tabular}


Table 2. Correlation between expression of NDR1 and clinical variables

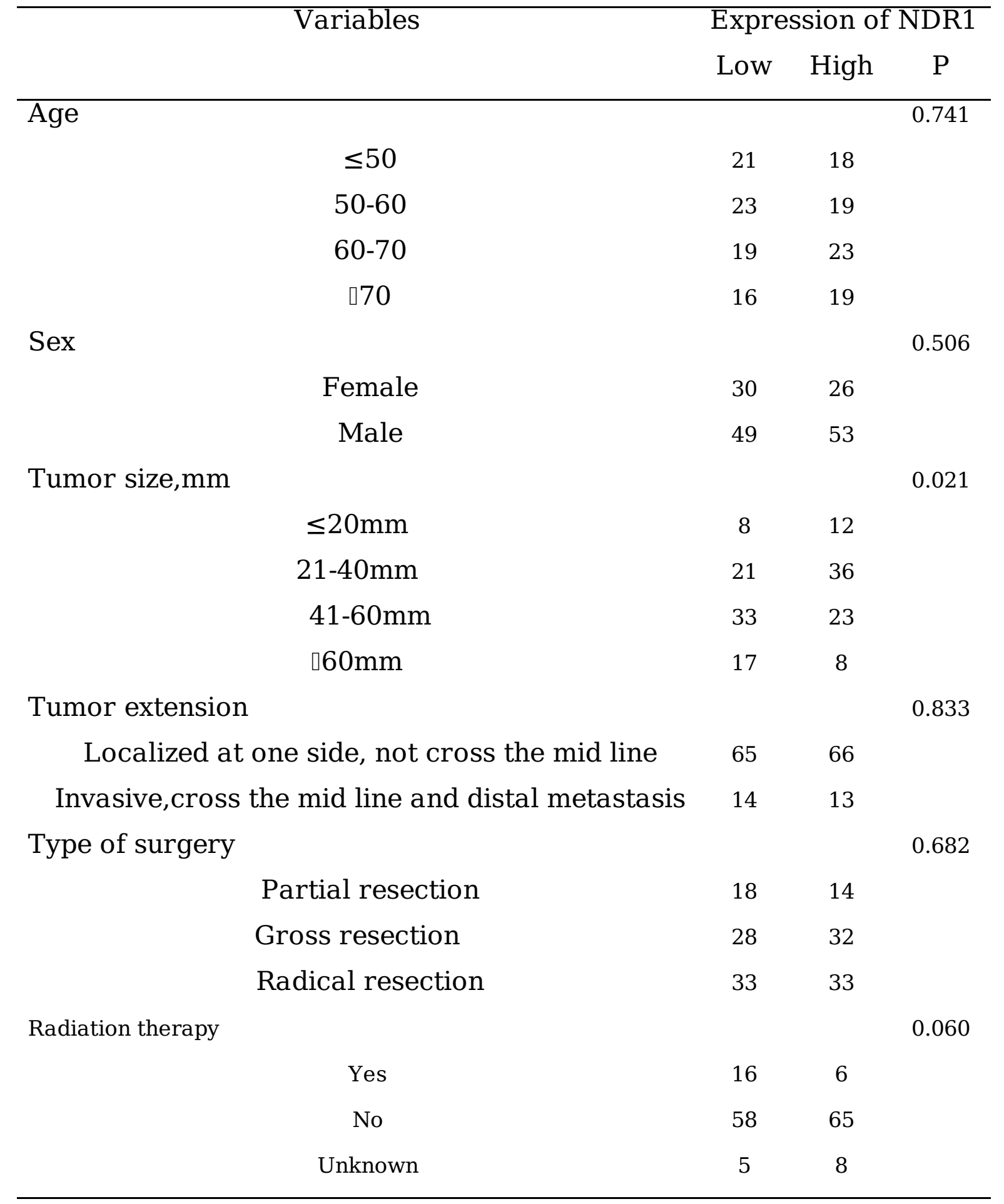


A

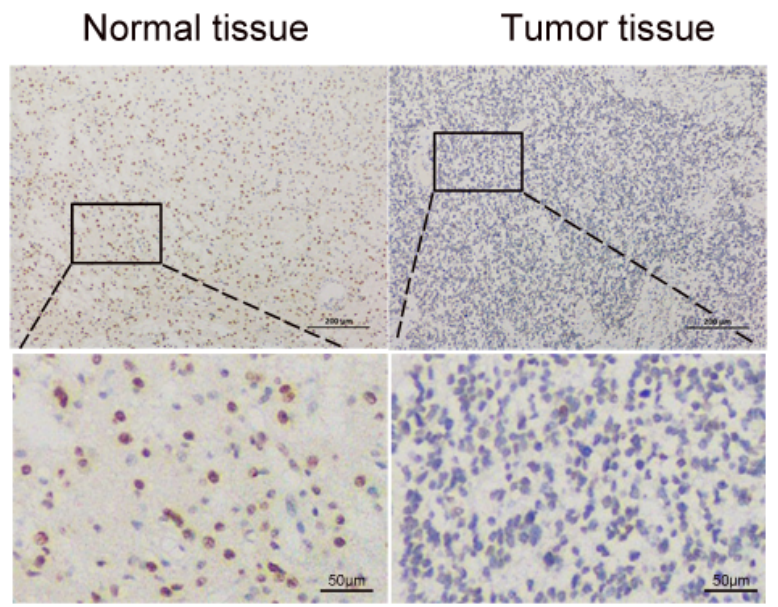

B

C
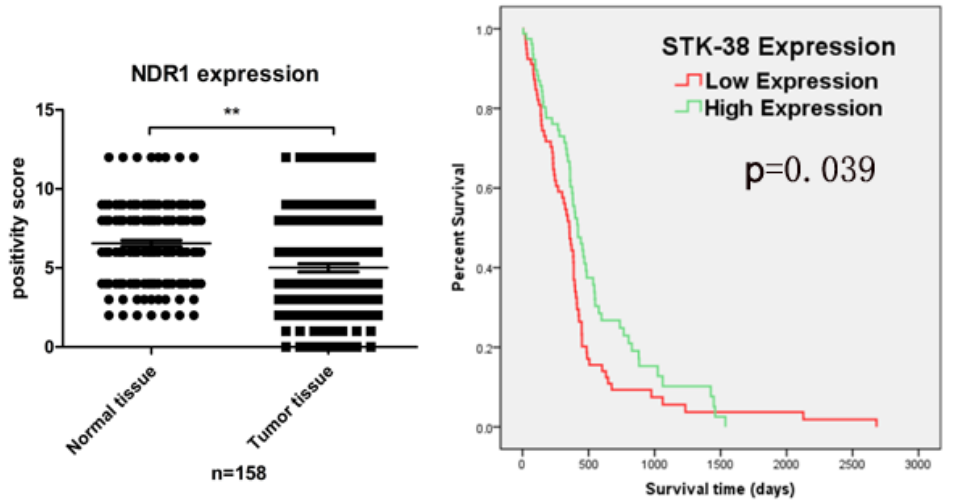

D

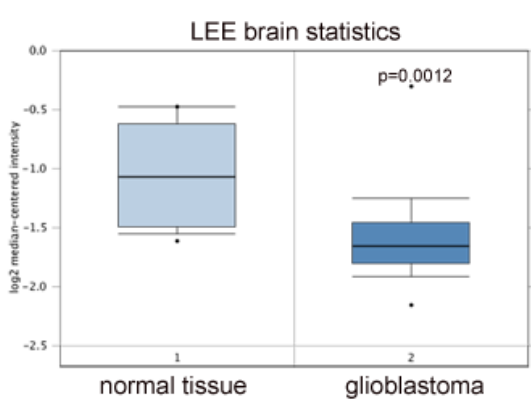

G

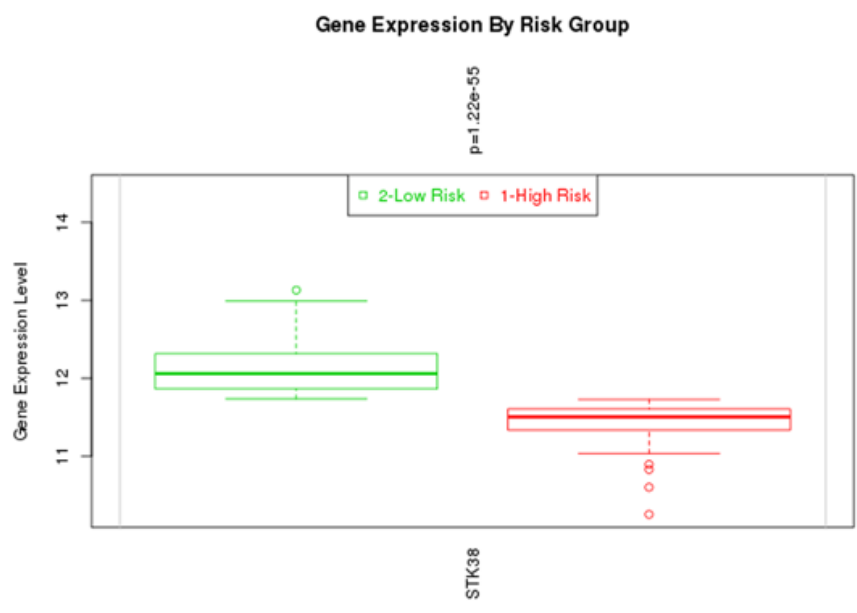

$E$

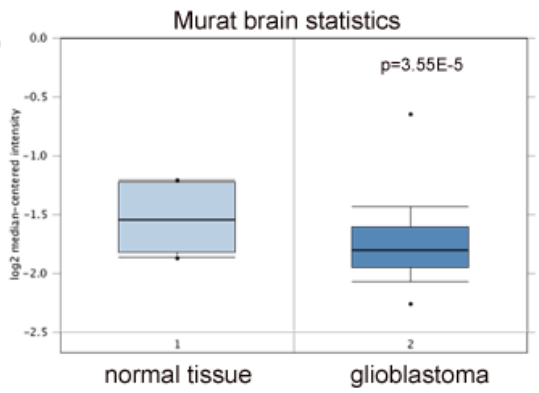

F

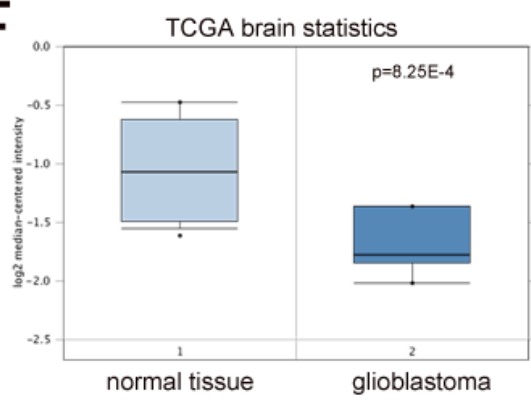

$\mathrm{H}$

CENSORED:SURVIVAL.MONTHS STK38
Concordance Index $=58.46$, Log-Rank Equal Curves $p=0.006528, R^{\wedge} 2=0.041 / 1$ Risk Groups Hazard Ratio $=1.42$ (conf. int. $1.1 \sim 1.84$ ), $p=0.006914$

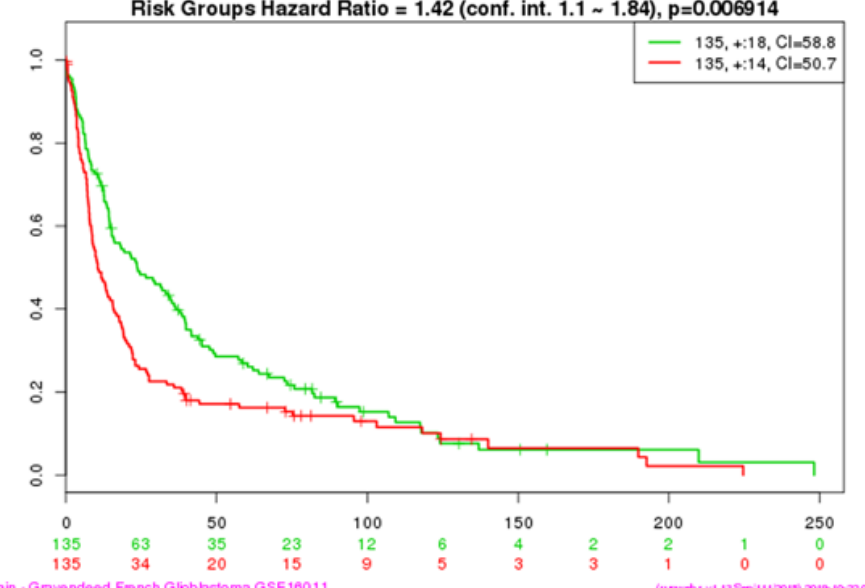

\section{Figure 1}

Expression of NDR1 is lower in GBM tissue than normal tissues. (A-B) The NDR1 protein level was lower in GBM tissue, as shown by immunohistochemical analysis $(n=158, \star \star P<0.05)$. (C) Association between the NDR1 level and overall survival $\left(n=158\right.$, $\left.{ }^{*} P<0.05\right)$. (D-F) Low mRNA expression of NDR1 in GBM tissues (ONCOMINE database) ( $* * P<0.05)$. (G) Gene expression of NDR1 divided by risk (SurvExpress dataset) $\left({ }^{\star *} \mathrm{P}<0.05\right)$. $(\mathrm{H})$ Censored survival months of NDR1(SurvExpress dataset) $\left({ }^{\star} \mathrm{P}<0.05\right)$. (I) Mean score of NDR1 of tumor tissues and normal tissues of GBM patients. 
A

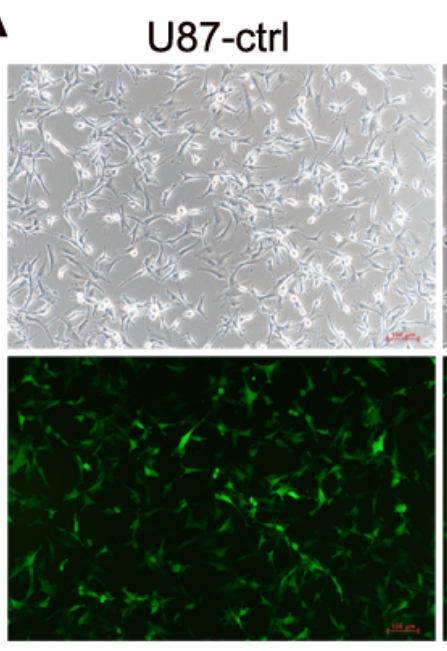

B

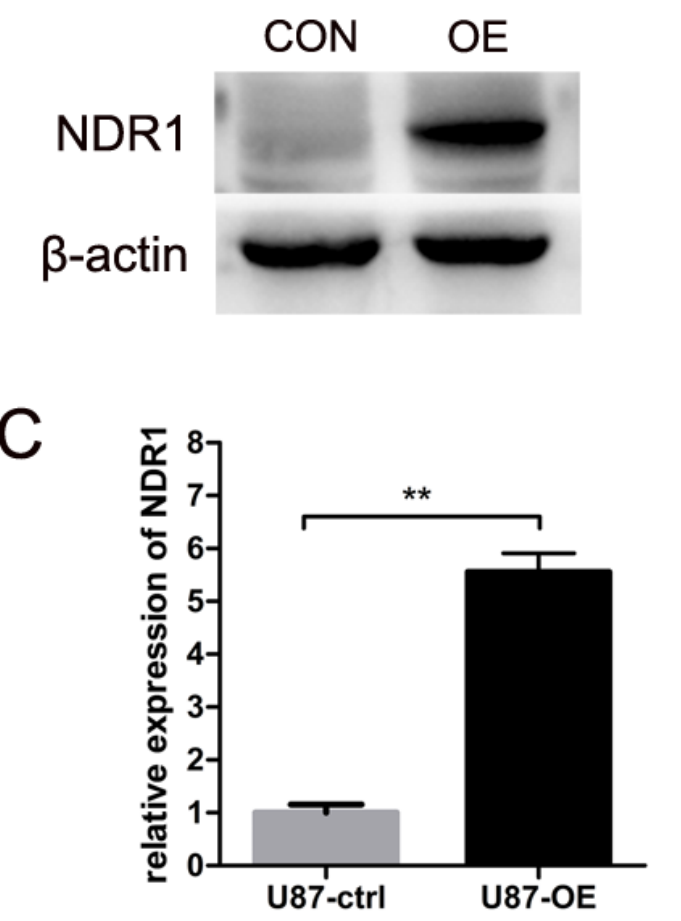

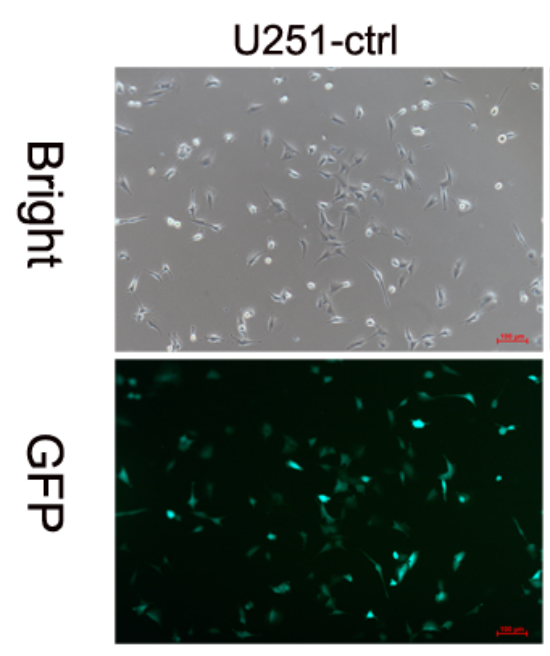
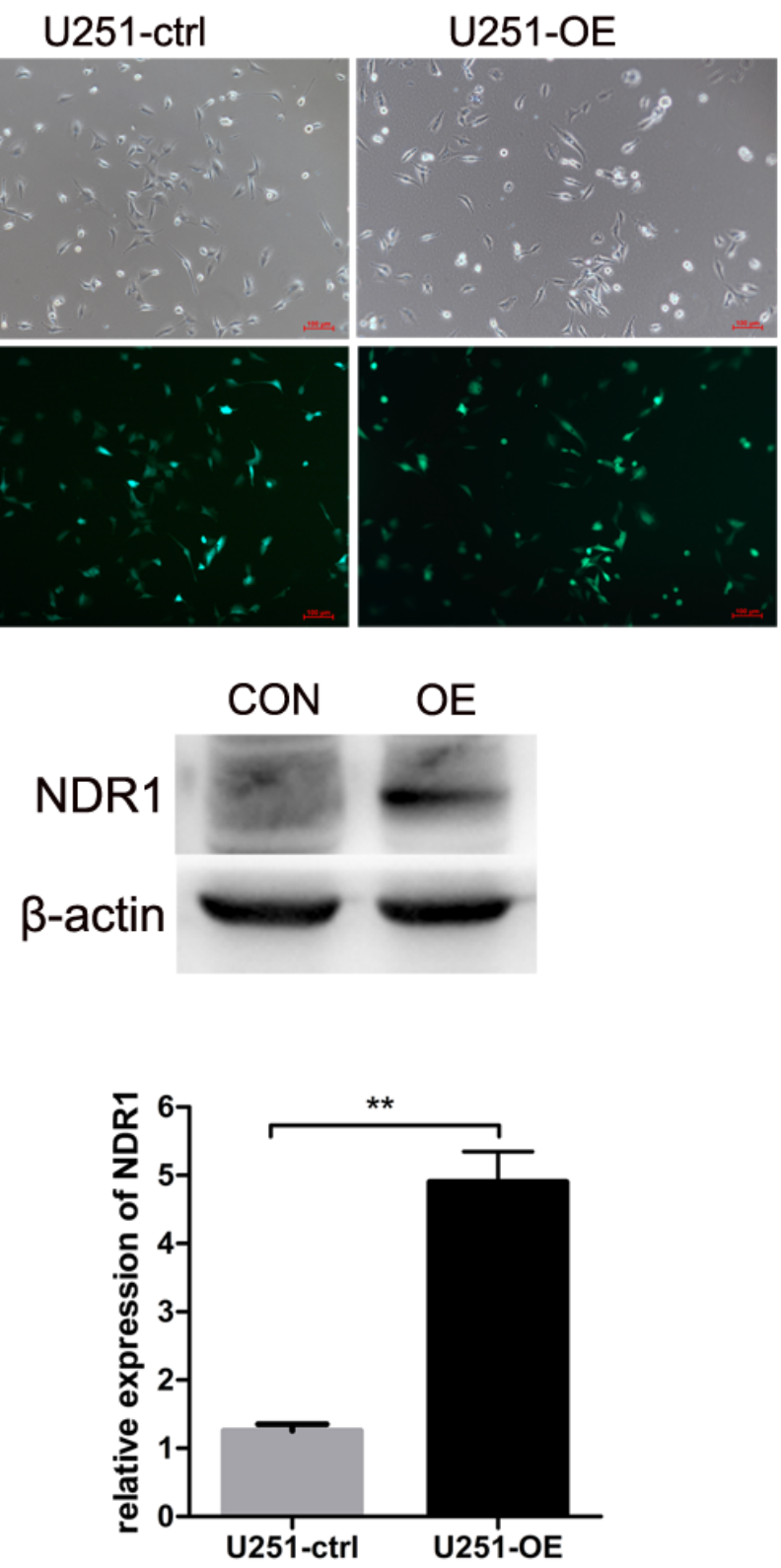

\section{Figure 2}

Lentivirus-mediated over-expression upregulated the expression of NDR1 in U87 and U251 cells. (A) The GBM cancer cells are examined by fluorescence microscopy after lentivirus infection for $72 \mathrm{~h}$. (B) The protein levels of NDR1 are upregulated in U87 and U251 cells infected with Lv-OE-NDR1. Every result is verified at least three times. (C) Real-time qPCR indicates mRNA levels are higher in U87 and U251 cells infected with Lv-OE-ctrl than Lv-OE-NDR1. (**p < 0.05). 

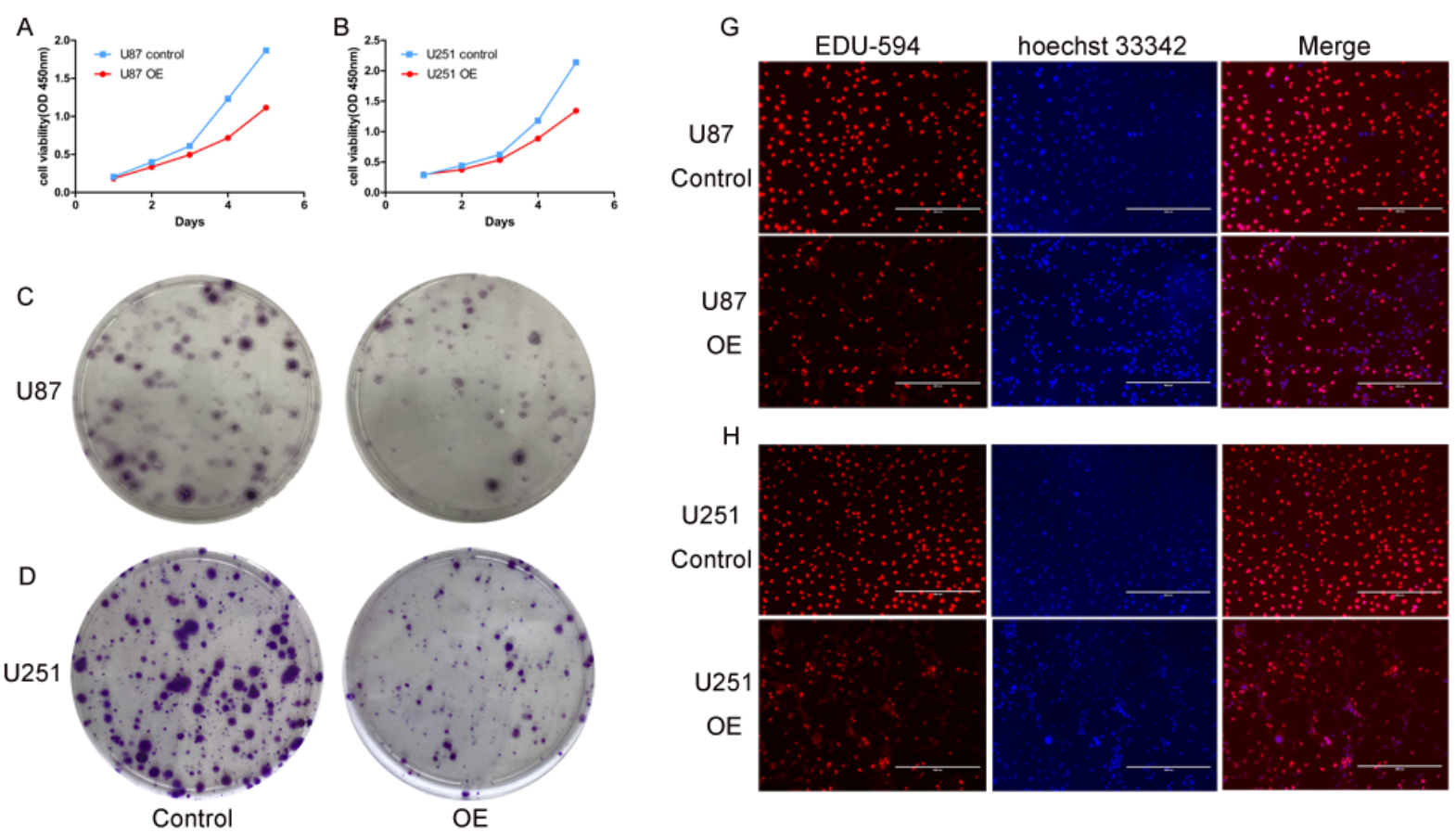

E
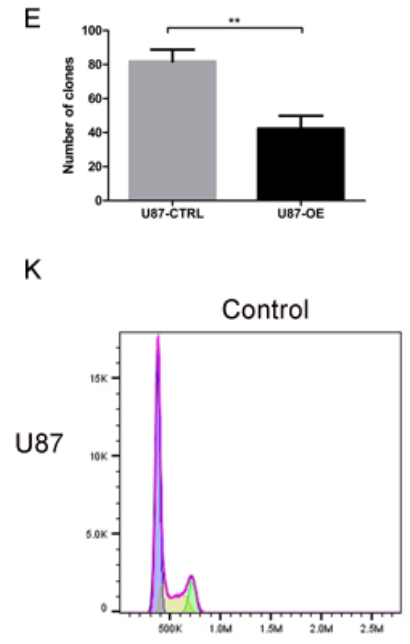

FL10-A: PE-A

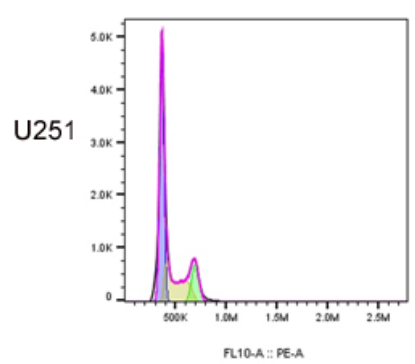

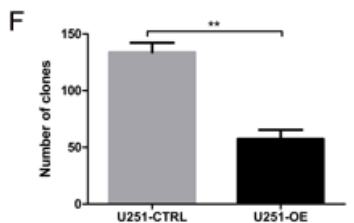

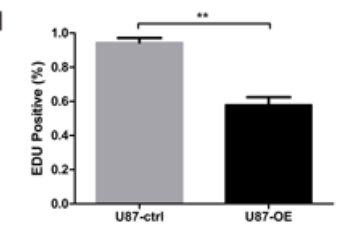

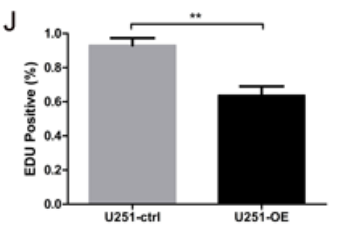

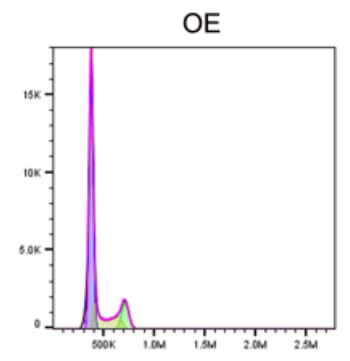

PL10-A : PE-A

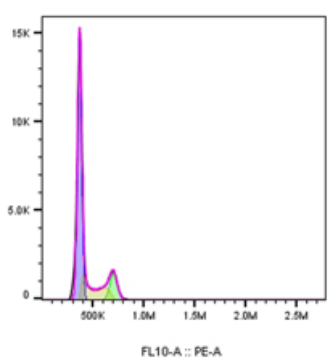

$\mathrm{L}$
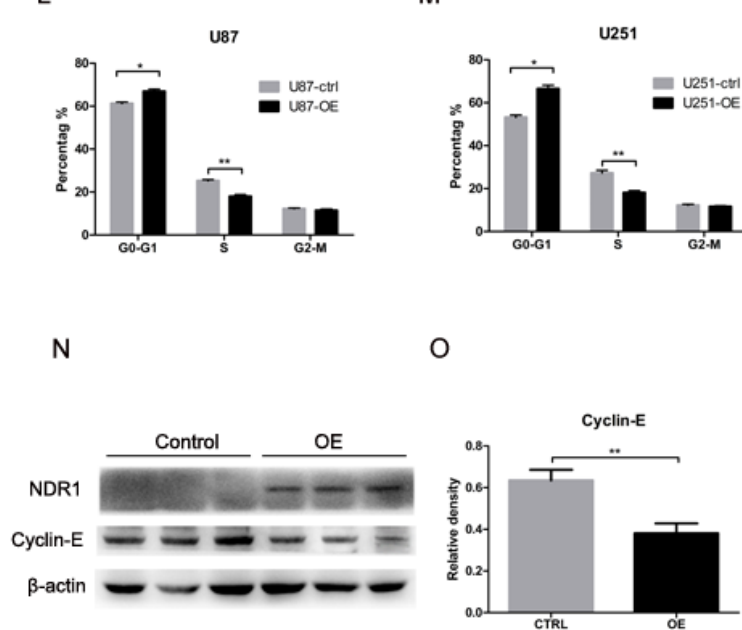

\section{Figure 3}

NDR1 overexpression supressed cell proliferation in vitro. (A,B) Cell viability of U87 and U251 control group and overexpression group were validated by CCK-8 assay. (C-F) Number of cell clones of U87 and U251 control group and overexpression group, **P®0.05. (G-J) EDU immunofluorescence images of U87ctrl and U87-OE. In NDR1-OE group, the number of EDU positive cells was reduced when compared to NDR1-ctrl group (**P囚0.05). (K-M) cell cycle analysis result of U87-ctrl and U87-OE groups, U251-ctrl and 
U251-OE groups. NDR1 overxepression significantly arrested cell cycle at G1 phase with a concomitant reduction in proportion of $S$ phase ( ${ }^{\star * P ख 0.05) . ~}(\mathrm{~N}, 0)$ Western blotting results of cyclin-E1 and NDR1. In NDR1-OE group, the expression of cyclin-E1 was reduced when compared to NDR1-ctrl group (**P囚0.05).

A

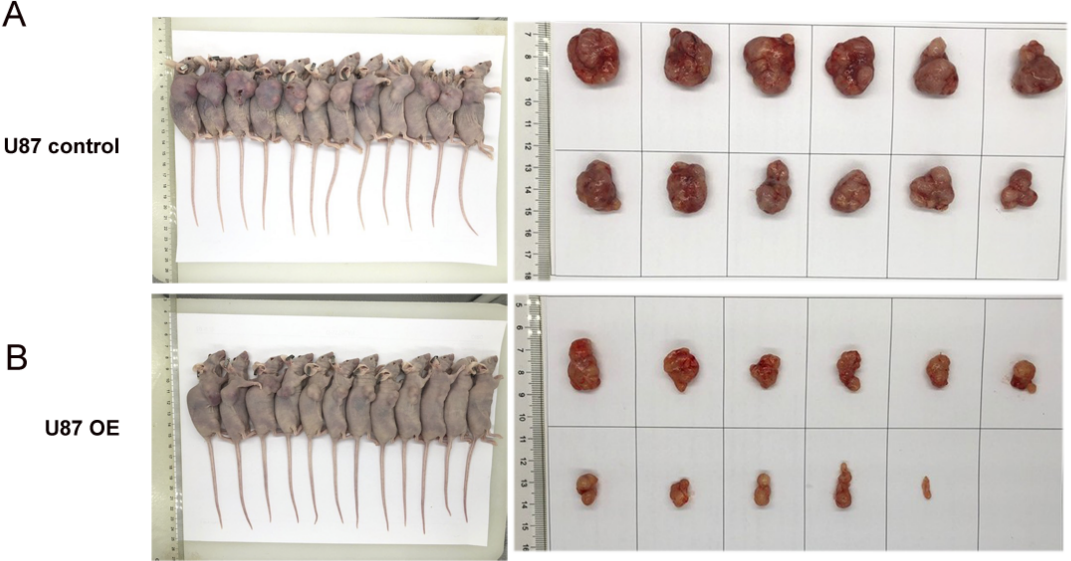

C

U87 contro NDR1
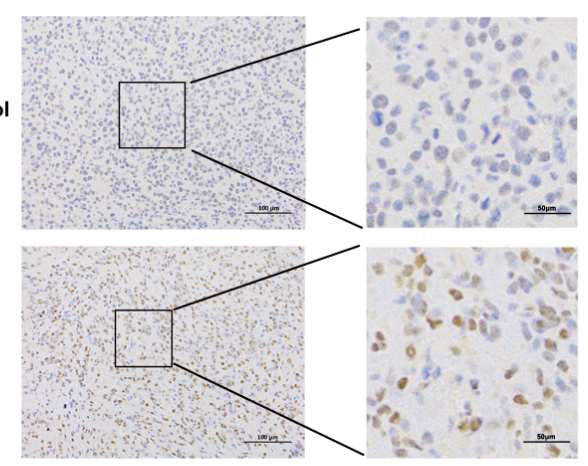

$\mathrm{F}$

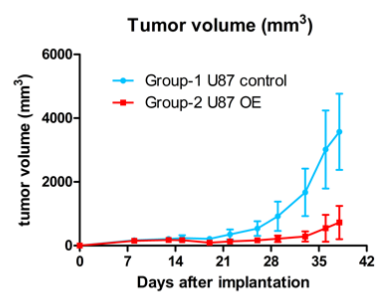

$\mathrm{D}$

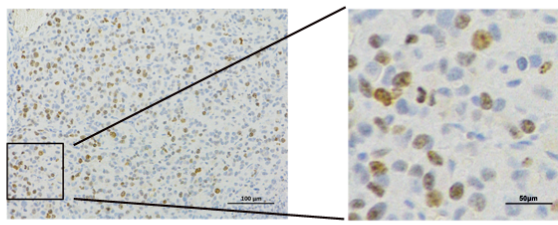

G

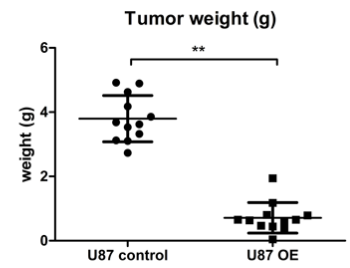

$\mathrm{H}$

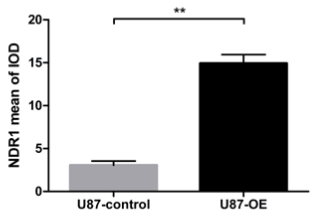

$E$
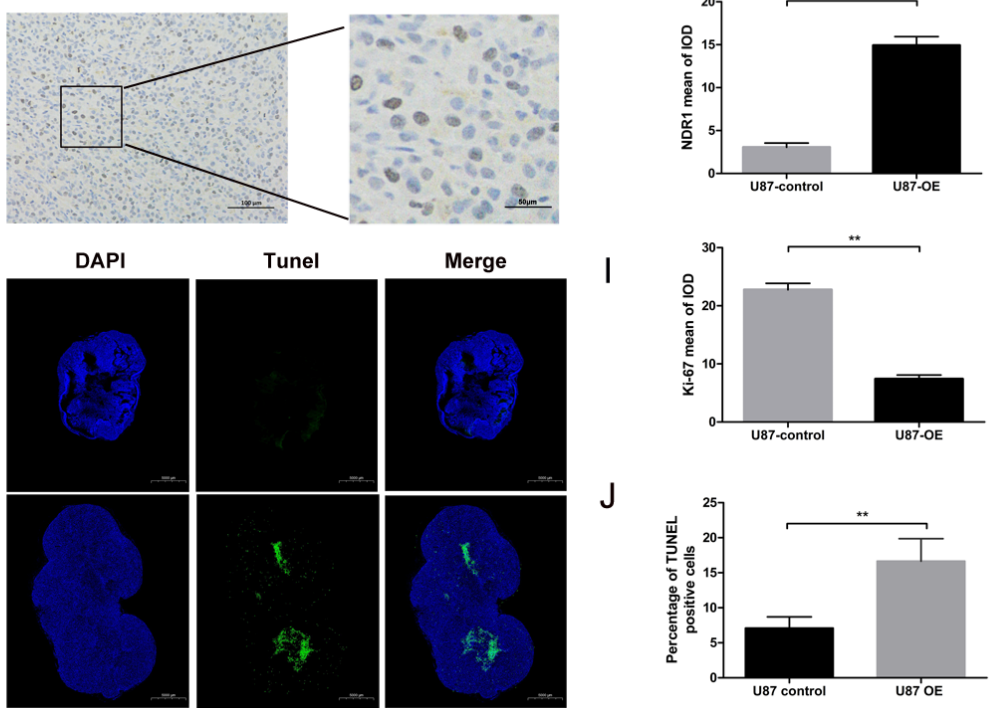

U87 control
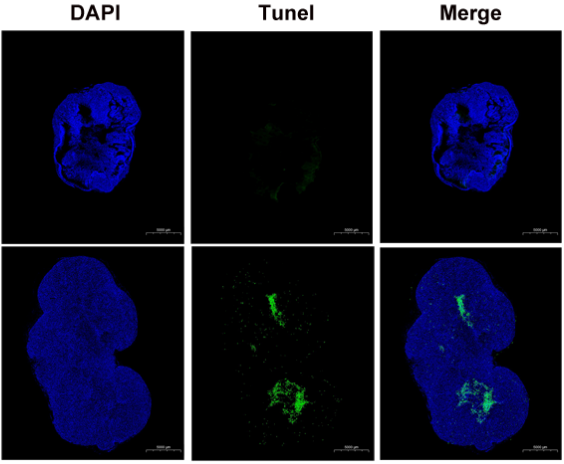

Figure 4

NDR1 overexpression inhibited tumor growth in vivo. $(A, B)$ Representative picture of animals and xenograft tumors. (C) Representative expression of NDR1 of xenograft from U87-ctrl and U87-OE group. 
(D) Representative expression of Ki-67 from U87-ctrl and U87-OE group. (E) Terminal Deoxynucleotidyl Transferase Deoxyuridine Triphosphate Nick-End Labeling Assay (TUNEL) images of U87-ctrl and U87OE. (F-G) statistical results of tumor volume and tumor weight $(B, C)$ statistical results (**P囚0.05).. (H-J) statistical results of NDR1, Ki-67 and TUNEL staining(B,C) statistical results (**P囚0.05).
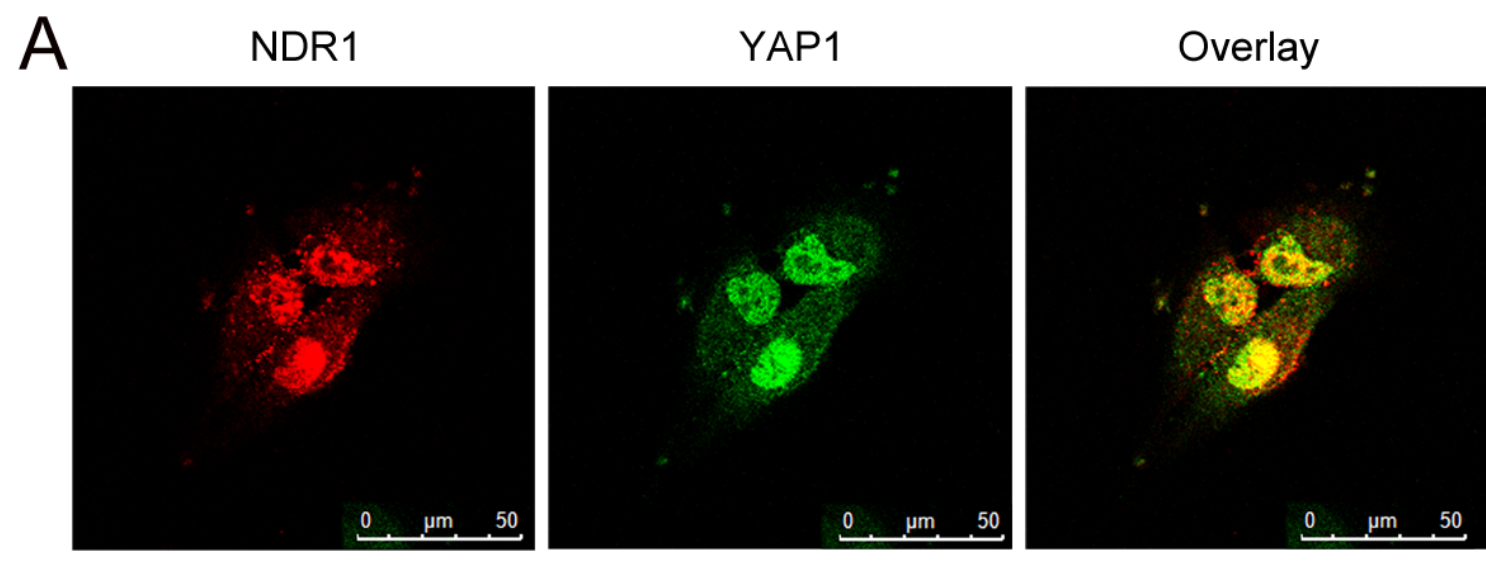

B

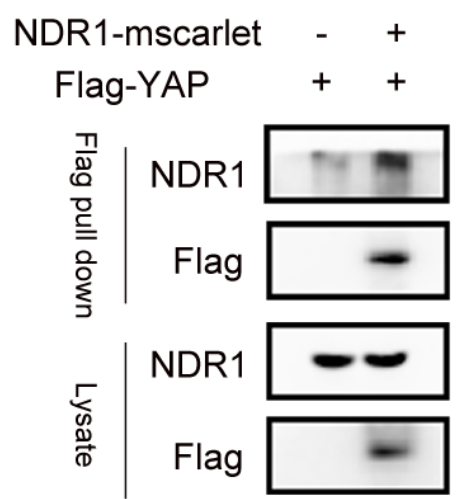

D
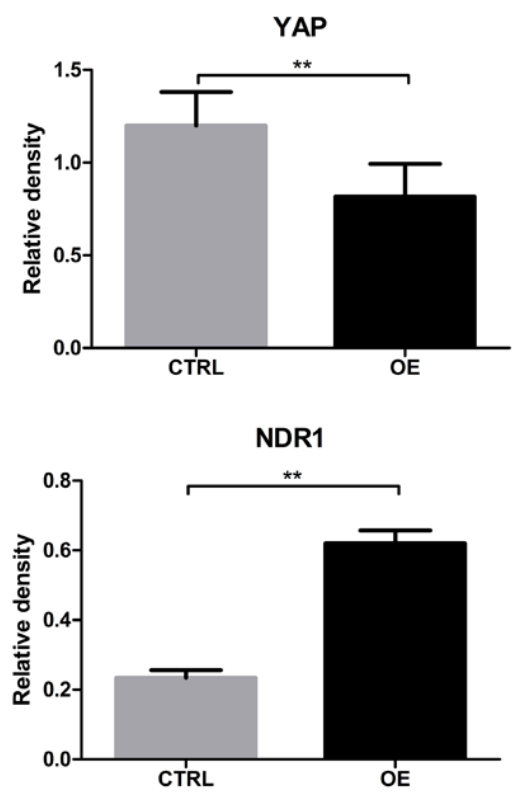
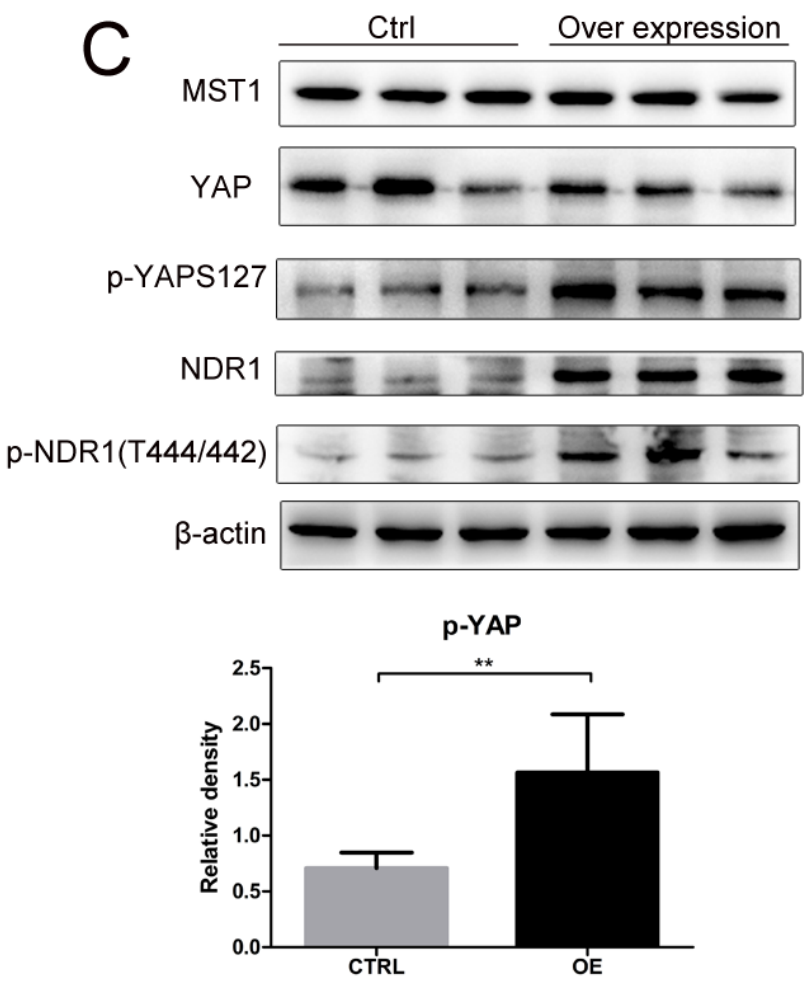

p-NDR1

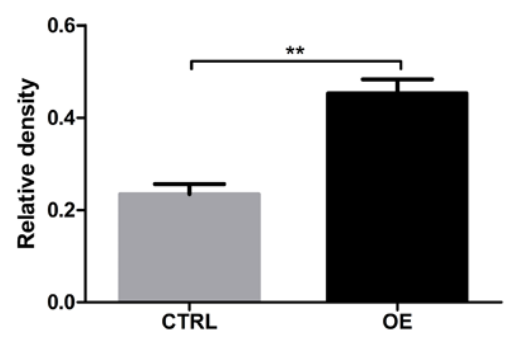

Figure 5 
NDR1 phosphorylate YAP on Ser127. (A) Immunofluorence microscopy images of NDR1 and YAP. The overlap image showed that NDR1 significantly colocalizes with YAP. (B) Co-IP results of NDR1 and YAP. Results showed that NDR1 could interact with YAP. (C,D,E,F,G) Endogenous protein levels of phopho-127 YAP, total YAP, MST1, NDR1 and p-NDR1 (T444/442) and $\beta$-actin were examined by western blotting (**P区 0.05).

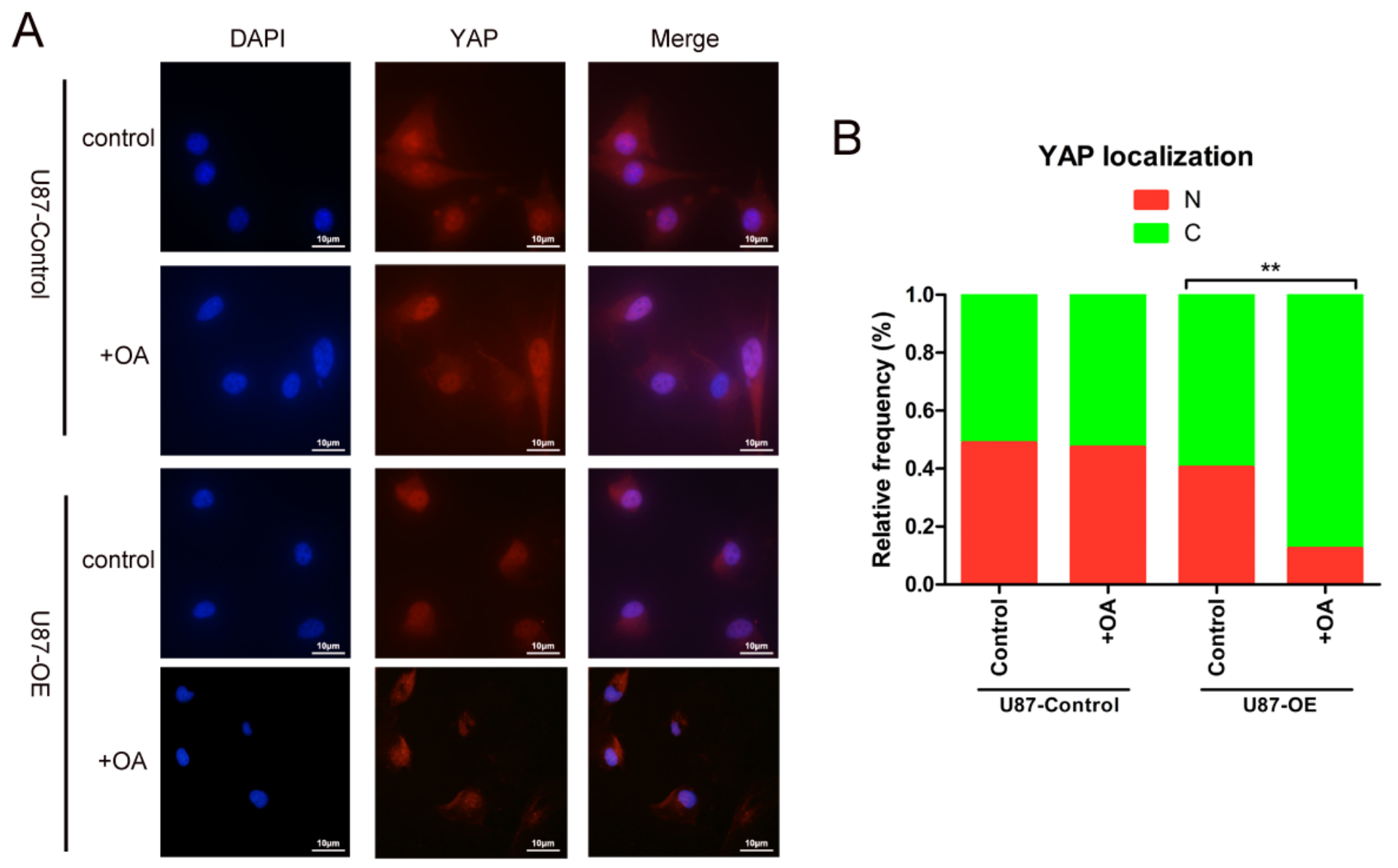

\section{Figure 6}

NDR1 regulates cellular distribution of YAP. (A) cellular distribution of endogenous YAP in U87-ctrl and U87-OE in Okadaca acid (OA) inducible manner. When stimulated with OA, more YAP were restrained in cytoplasm. (B) quantification of the nuclear/cytoplasmic YAP ratio per cell. (**p囚0.05). 

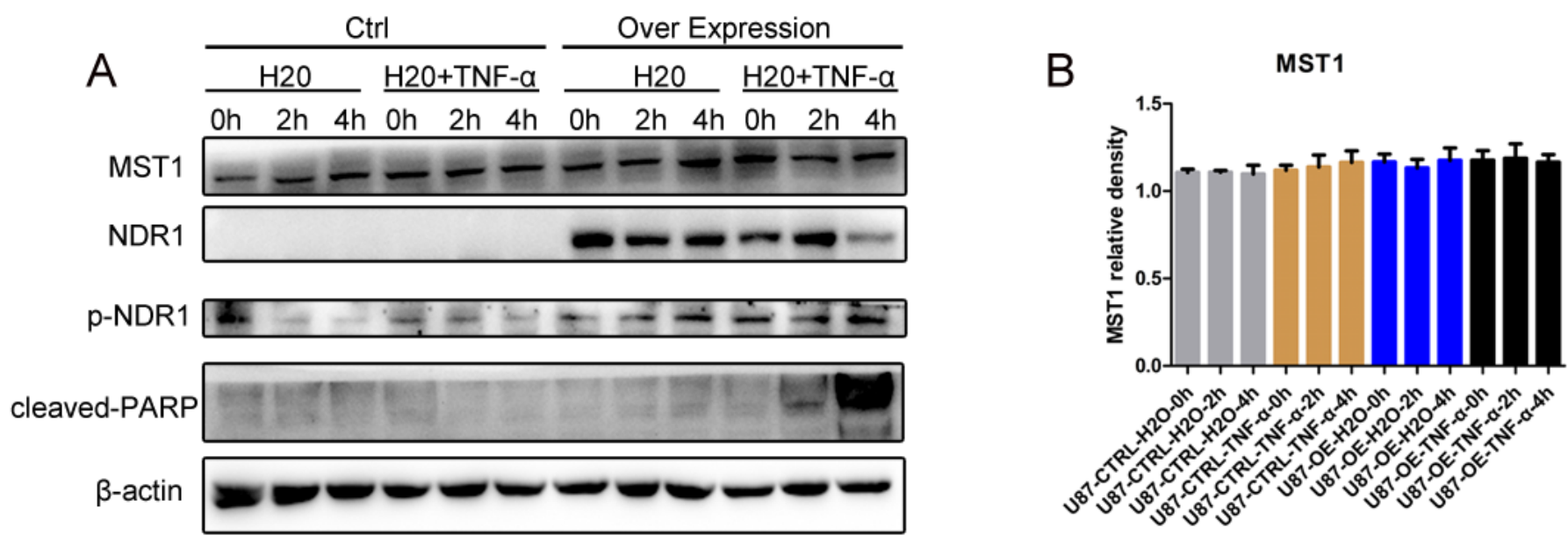

C

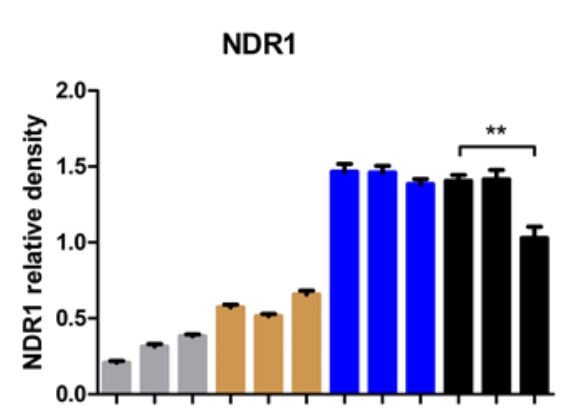

D

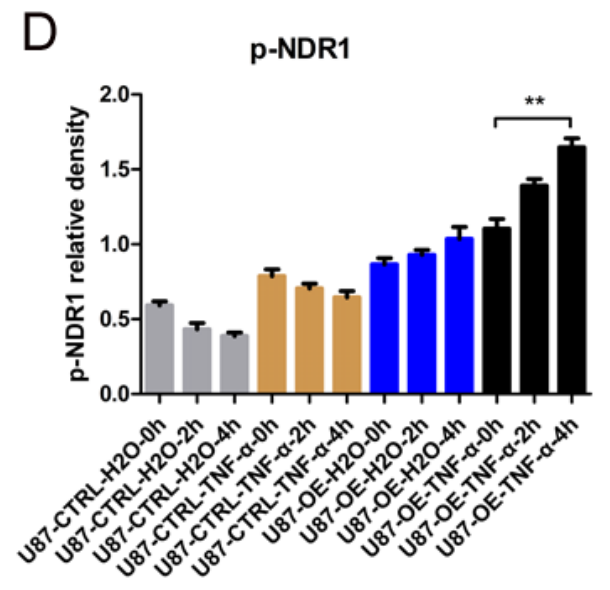

E
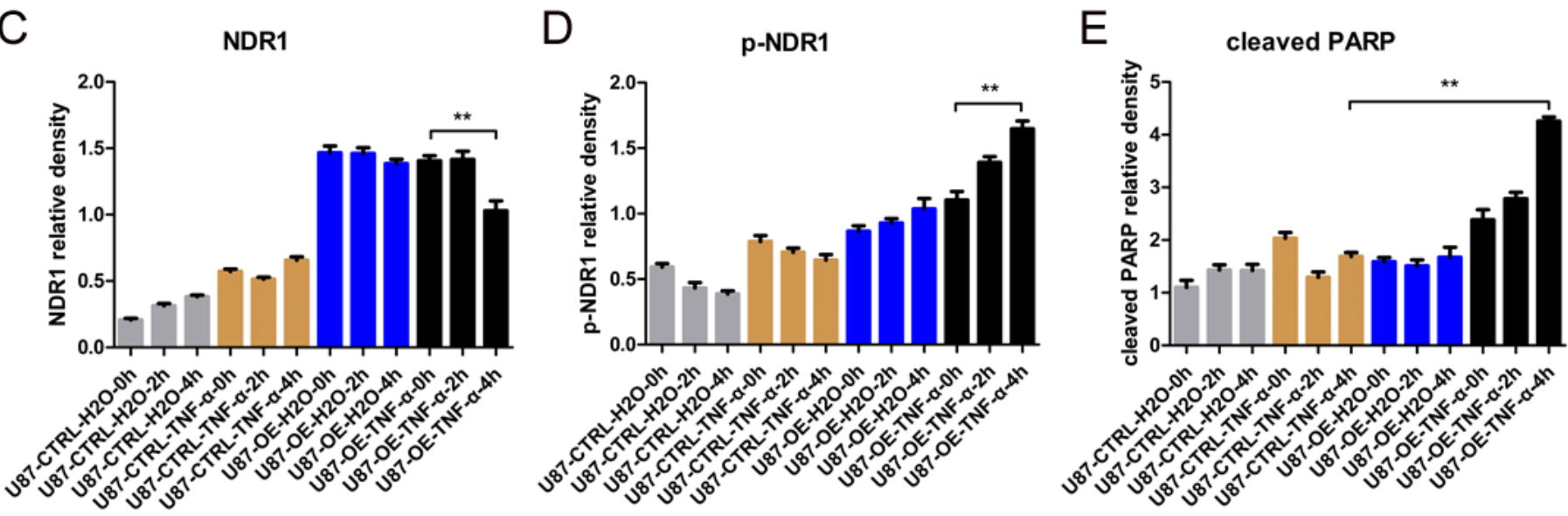

Figure 7

NDR1 mediates cell apopptosis. (A) U87-ctrl and U87-OE cells were stimulated with or without $\mathrm{H} 2 \mathrm{O}$ and TNF- $a$ for $0 h, 2 h$ and $4 h$. The protein level of MST1, NDR1 and p-NDR1(T444/442), cleaved PARP and $\beta$ actin were examined by western blotting. (B-E) statistical results (**P®0.05). 


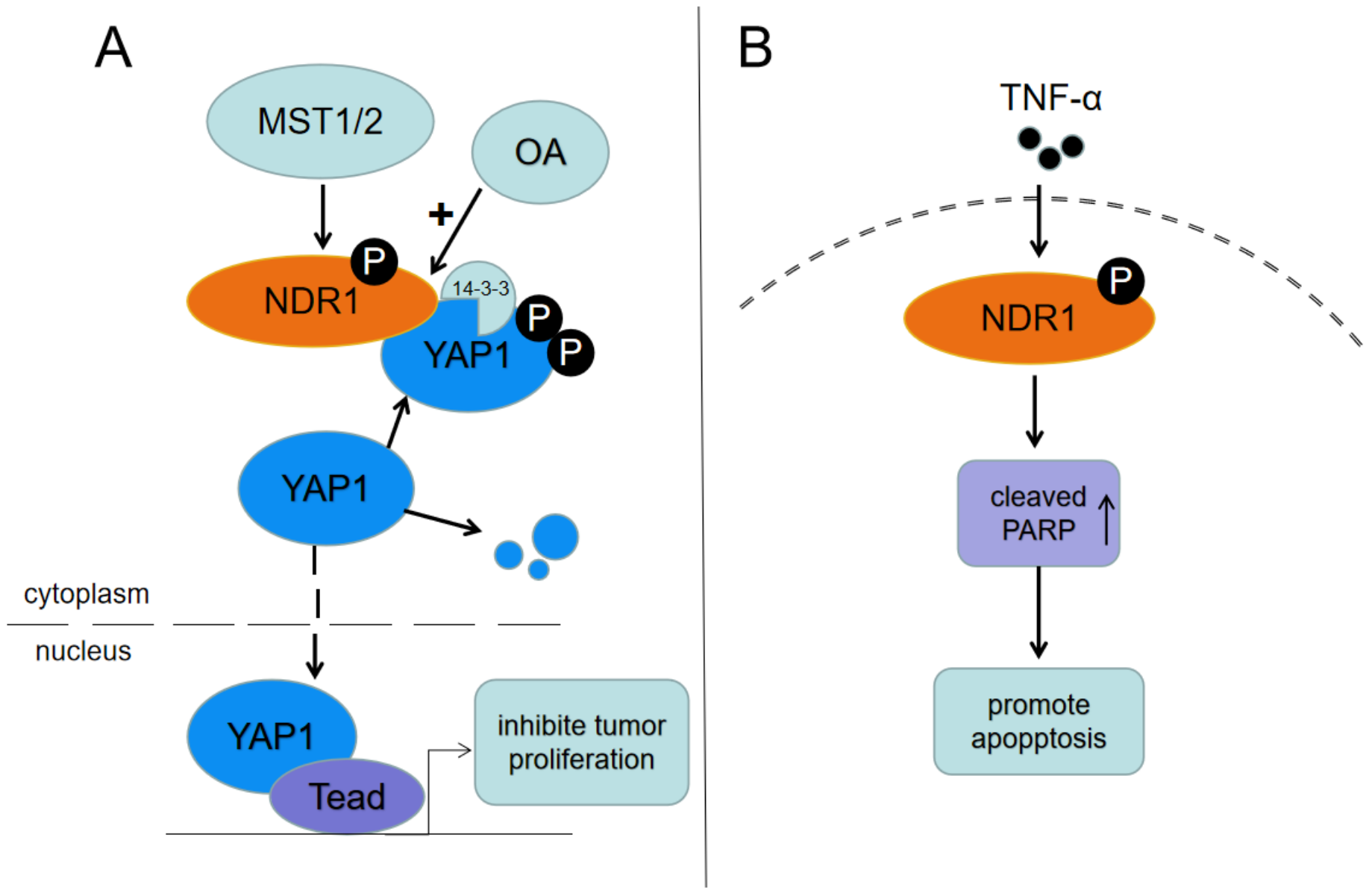

Figure 8

Chart of proposed signaling pathway for NDR1 and YAP.

\section{Supplementary Files}

This is a list of supplementary files associated with this preprint. Click to download.

- Supplementarymaterials.docx

- renamed0cbb6.tif

- renamed750be.tif

- renamed7eba3.tif 